\title{
Protein Coding Assignment of the S Genes of the Avian Reovirus S1133
}

\section{Thomas J. Schnitzer}

\author{
Rackham Arthritis Research Unit, Department of Medicine, University of Michigan School of Medicine, \\ Ann Arbor, Michigan 48109
}

Received September 4, 1984; accepted October 30, 1984

\begin{abstract}
The protein coding assignments for each of the $\mathrm{S}$ genes of the avian reovirus $\mathrm{S1133}$ have been determined. In vitro translation of RNA derived from individual S dsRNA genome segments demonstrated that the largest $\mathrm{S}$ gene, $\mathrm{S1}$, codes for the smallest protein, $\sigma_{C}$; the $\$ 2$ for $\sigma_{A}$; the $S 3$ gene for $\sigma_{C}$; and the $S 4$ gene for $\sigma_{N S}$. No other gene products could be identified. By examination of appropriate reassortant viruses, these assignments were confirmed. (c) 1985 Academic Press, Inc.
\end{abstract}

Although the avian reoviruses demonstrate differences from the mammalian reoviruses in biological and serological properties, they share many morphological and physico-chemical characteristics which have resulted in their classification into the same group of viruses (1-5). Recent biochemical studies $(1,3,6-8)$ have confirmed the basic genetic and structural similarities between these two groups of viruses. Both contain a genome consisting of 10 segments of double-stranded (ds) RNA separable into three size classes. Each gives rise to 10 distinct viral-specified products, eight of which are found in the complete, infectious virion. Furthermore, the arrangement and relative quantities of these structural proteins also appear to be quite similar.

In the mammalian reoviruses, a series of investigations $(9,10)$ has resulted in the assignment of each of the individual viral genes to its respective protein product. This has been accomplished by two complimentary approaches: (1) in vitro translation of individual viral + strand RNAs and (2) analysis of the polypeptide and genome patterns upon polyacrylamide gel electrophoresis (PAGE) of appropriate reassortant viruses. Using similar biochemical and genetic techniques, studies in this laboratory have now enabled determination of the protein coding assignments of each of the four $S$ genes of the avian reoviruses.
In these investigations the S1133 and Lasswade strains of avian reovirus (7) were utilized. For the in vitro translation studies, the S1133 virus was grown in chick embryo fibroblasts (CEF) and purified by repetitive cesium chloride equilibrium gradient centrifugation as previously described (8). Viral dsRNA was then extracted and run on a 5\% Tris-acetate polyacrylamide gel. dsRNA segments were visualized after staining with ethidium bromide and the gel was cut to permit separation of individual S gene segments. The dsRNA was removed from the gel by electroelution and then extracted twice with phenol:chloroform:isoamyl alcohol and once with chloroform before being ethanol precipitated overnight. Prior to use, each of the RNA preparations was quantitated and appropriate aliquots melted in $90 \%$ DMSO for $30 \mathrm{~min}$ at $50^{\circ}$. The separated RNA strands were precipitated and resuspended in the translation mixture at the time of use. The translation protocol consisted of the standard BRL rabbit reticulocyte in vitro translation system modified by adjusting the final potassium $(\mathrm{K}+)$ ion concentration to 40 $\mathrm{m} M$ with $\mathrm{KAc}$, this concentration giving optimal $\left[{ }^{35}\right.$ S $]$ methionine incorporation. For most experiments, $5 \mu \mathrm{g}$ of dsRNA were utilized per reaction mixture. Incorporation of radioisotope was found to be linear in this range, declining at higher RNA concentrations. The reaction was allowed 
to proceed at $30^{\circ}$ for $60 \mathrm{~min}$ and then terminated by placing on ice. Aliquots were precipitated with TCA to determine total incorporation and the remainder subjected to immune precipitation utilizing rabbit antisera to $\mathrm{S1133}$ as has been previously described (9). This material was then analyzed by electrophoresis on standard 9\% polyacrylamide gels followed by fluorography.

Figure 1 demonstrates the products obtained upon in vitro translation of each of the $S$ genes of the avian reovirus $S 1133$. To permit identification of each band, an immunoprecipitate from a lysate of $\left[{ }^{35} \mathrm{~S}\right]$ methionine-labeled cells previously infected with S1133 was run in parallel in all experiments. As can be seen, the $S 1$ gene, the largest of the $S$ genome segments, gave rise to the smallest reovirus protein, $\sigma_{\mathrm{C}}$. The possibility of an additional protein product, either a primary translational product or a cleavage product, was considered but no evidence of any smaller molecular weight polypeptides was found. The $\mathrm{S} 2$ gene product was shown to comigrate with the $\sigma_{\mathrm{A}}$ protein band from S1133. In vitro translation of the $\mathrm{S} 3$ gene occasionally produced two bands when analyzed as described, the major incor-

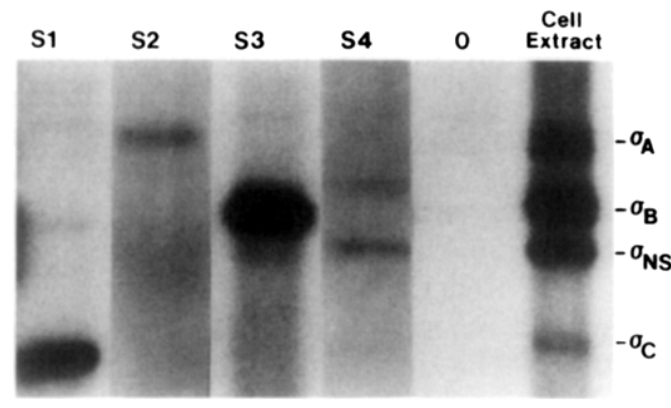

FIG. 1. SDS-polyacrylamide gel analysis of rabbit reticulocyte cell-free protein synthesizing system programmed with fractionated DMSO-treated $S_{1}$, $S_{2}, S_{3}$, and $S_{4}$ genome segments of avian reovirus S1133. [ $\left.{ }^{35} \mathrm{~S}\right]$ Methionine-labeled products were immunoprecipitated and compared by PAGE analysis with $\left[{ }^{25}\right.$ S]methionine-labeled viral protein immunoprecipitated from S1133-infected cells (cell extract). Lane $O$ represents immunoprecipitated product obtained when the translation system was programmed with only globin mRNA. poration occurring in a band with mobility of the $\sigma_{B}$ reovirus protein. In addition, a less dense band migrating in the position of $\sigma_{\mathrm{NS}}$ was often found in these preparations. As the $\sigma_{\mathrm{NS}}$ protein could be shown to be the product of the S4 gene, the possibility that the S3 dsRNA was contaminated by a minor amount of $\mathrm{S} 4$ dsRNA was examined by re-running the S3 dsRNA on a second polyacrylamide gel after it had been electroeluted from the first gel. Invariably a small amount of RNA, detectable by ethidium bromide staining, could be seen to migrate in the position of the S4 dsRNA band. Prior treatment of extracted total viral RNA with proteases, or the use of higher SDS concentrations, failed to prevent this apparent "sticking" of S4 dsRNA to the S3 dsRNA.

In order to confirm these protein coding assignments, a second independent approach was undertaken. Reassortant viruses were produced by dual infection of CEF with both the S1133 and Lasswade strains of avian reovirus. Previous studies $(6,7)$ had demonstrated that two of the four S dsRNA genome segments of these two viruses could easily be differentiated by significant differences in their mobility upon PAGE. A third S genome segment of each virus (S3 of Lasswade and S4 of S1133) comigrated, but assignment of this band could be made to either parental virus by assuming that any viable reassortant would contain one of each of the $S$ genes rather than two copies of the same gene. The S1 genes of each of these viruses also comigrated but could not be differentiated. Thus, the parental origin of three of the four $S$ genes in the reassortant viruses could be unequivocally determined.

Figure 2A depicts the pattern of migration of the dsRNA genome segments for a group of reassortant viruses produced using S1133 and Lasswade as the parental strains. In those instances in which there is comigration of the dsRNA genome segments (indicated by the dashed line), the parental origin, determined as described above, has been indicated. No assignment of $\mathrm{S} 1$ genes has been made. 

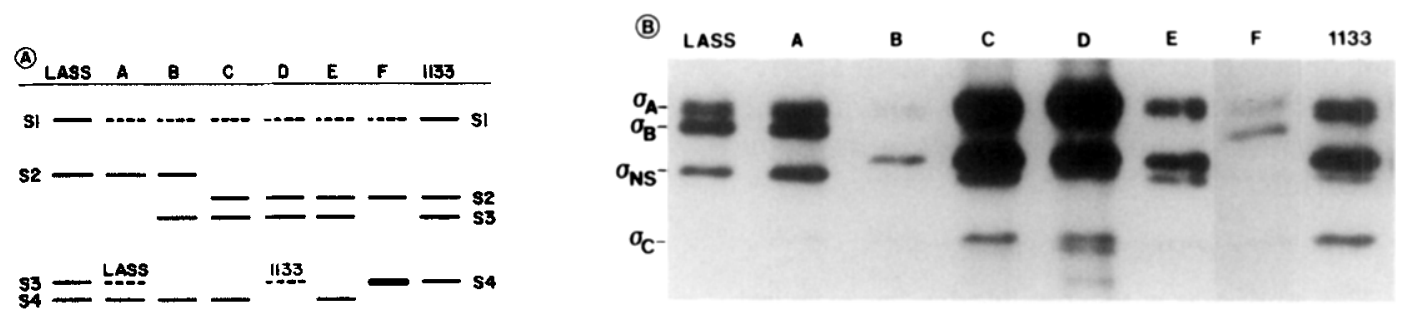

FIG. 2. (A) Diagrammatic representation of the $S$ dsRNA patterns observed upon PAGE of viral RNA obtained from the $\$ 1133$ and Lasswade avian reovirus and six selected reassortants (A-F). The dashed lines indicate those genes for which parental origin could not be definitely determined by mobility alone. (B) SDS-PAGE analysis of $\left[{ }^{35}\right.$ S $]$ methionine-labeled proteins obtained by immunoprecipitation from cells infected with $\mathrm{S} 1133$ and Lasswade strains of avian reovirus as well as each of the reassortant avian reoviruses $(\mathrm{A}-\mathrm{F})$ whose $\mathrm{S}$ genome pattern is shown in Fig. 2A.

Figure 2B demonstrates the pattern of migration upon PAGE of the immunoprecipitated [ $\left.{ }^{35} \mathrm{~S}\right]$ methionine-labeled viral proteins obtained from lysates of CEF infected with each of the reassortant viruses shown in Fig. 2A. Examination of this figure demonstrates that two bands, those corresponding to the viral proteins $\sigma_{\mathrm{B}}$ and $\sigma_{\mathrm{NS}}$, can clearly be distinguished among the reassortant viruses. Reassortant virus $A$ and $F$ alone contain a $\sigma_{B}$ band that is significantly retarded compared to those seen for reassortant viruses B, C, D, and E. Reassortants A and F both contain the S3 gene from Lasswade; additionally, the mobility of the $\sigma_{\mathrm{B}}$ protein band of these viruses is found to be identical to that of the $\sigma_{\mathrm{B}}$ band of Lasswade when examined under similar conditions. In the case of the $\sigma_{\mathrm{NS}}$ band, reassortants $D$ and $F$ both demonstrate a band with mobility retarded relative to the same band seen in the other reassortants examined, and they alone have the $\mathrm{S} 4$ gene from S1133. Again, the comigration of this band with that of the parental S1133 has been confirmed by simultaneous PAGE of radiolabeled protein from each virus. Because of the fact that both the $\sigma_{A}$ and $\sigma_{C}$ proteins of the two viruses could not be differentiated upon PAGE, it was not possible to confirm the results of the in vitro translation studies by this technique for the S1 and S2 genes.

Thus, these studies have defined the protein coding assignments for each of the four S genes of the S1133 avian reo- virus: $S 1$ codes for $\sigma_{C}, S 2$ for $\sigma_{A}, S 3$ for $\sigma_{B}$, and $S 4$ for $\sigma_{N S}$. Because the relative molecular weights of the $S$ genome segments and the $\sigma$ proteins have been assumed to vary in direct relation to their mobility upon PAGE, it was unexpected to find the supposedly largest gene, S1, coding for the lowest molecular weight $\sigma$ protein, $\sigma_{\mathrm{C}}$. The relative molecular weights of the S2, S3, and S4 genome segments and their respective protein products were as might be expected.

Several explanations for the findings in regard to the S1 genome segment can be considered. First, it is possible that there is a second protein product produced from the S1 gene. Despite examination for such a lower molecular weight species, none has been found. Furthermore, in the mammalian reovirus system, in which all of the genes have been cloned (11), no evidence exists for such a situation. Second, and more likely, the mobility of either the dsRNA or the protein may not truly reflect its molecular weight but may be altered by secondary structures or other factors. Finally, there may be a silent or nontranslated portion of the S1 gene. This can only be determined after the gene has been fully sequenced.

One additional observation is the fact that there appears to be no detectable post-translational modification of the avian reovirus $\sigma$ proteins. The mobility upon PAGE of the products of in vitro translation comigrate with the true viral proteins obtained from infected cell ly- 
sates. This suggests, but obviously does not prove, that modifications, such as glycosylation or phosphorylation, do not occur with these avian reovirus proteins upon infection.

\section{ACKNOWLEDGMENTS}

This work was made possible by the technical assistance of Teresa Ramos and Karen Hassett. Support for this work was provided by a grant from the Kroc Foundation and from the United States Public Health Service (Research Grant RO1 AM27521 and Multipurpose Arthritis Center Grant AM20557 from the National Institutes of Health.) T. J. Schnitzer is a Senior Investigator of the Arthritis Foundation.

\section{REFERENCES}

1. JokLIK, W., Microbiol Rev. 45, 483-501 (1978).

2. OLson, N. O., “Diseases of Poultry," Chap. 26, pp. 641-647. (1978).
3. RAMig, R. F., and Fields, B. N., "The Molecular Biology of Animal Viruses," Chap. 8, pp. 383433. (1977).

4 VAN DER HeIde, L., Avian Pathol 6, 271-284 (1977).

5. Western Hemisphere Committee on Animal Virus Characterization, Amer. J. Vet. Res. 36, 861872 (1975).

6. Gouvea, V., and Schnitzer, T. J., J. Gen. Virol 61, 87-91 (1982).

7. GouveA, V., and Schnitzer, T. J., J. Virol 43, 465-471 (1982).

8. Schnitzer, T. J., Ramos, T., and GouveA, V., $J$. Virol. 43, 1006-1014 (1982).

9. MCCRAE, M. A., and JokLIK, W. K., Virology 89, 578-593 (1978).

10. Mustoe, T. A., Ramig, R. F., Sharpe, A. H., and FieldS, B. N., Virology 89, 594-604 (1978).

11. Cashdollar, L. W., Chmblo, R., Esparza, J., Hunson, G. R., and JokLIK, W. K., Virology 133, 191-196 (1984). 